11

\title{
Конфайнмент электрона в потенциале изображения и внешнем электростатическом поле
}

\author{
(C) П.А. Головинский ${ }^{1,2}$, М.А. Преображенский ${ }^{1}$ \\ ${ }^{1}$ Воронежский государственный технический университет, \\ Лаборатория фризических исследований, \\ 394006 Воронеж, Россия \\ ${ }^{2}$ Московский физико-технический институт (государственный университет), \\ 141701 Долгопрудный, Московская обл., Россия \\ e-mail: golovinski@bk.ru
}

Поступила в редакцию 01.12.2017 г.

В окончательной редакции 14.05.2018 г.

В квазиклассическом приближении установлено положение классических точек поворота при движении электрона, связанного полем изображения и постоянным однородным электрическим полем того же направления. Получены степенные разложения координат точек поворота в широком диапазоне энергий электрона и напряженностей поля. Описан механизм одномерного конфайнмента электрона, определяющего полностью дискретный спектр состояний. Установлена зависимость пространственной ширины области конфайнмента от напряженности поля и энергии электрона. Выполнены численные расчеты зависимости энергии электрона в разных состояниях от напряженности внешнего поля. Выполнено квазиклассическое квантование и определена зависимость энергии электрона от ширины области конфайнмента. Определен энергетический интервал максимальной плотности состояний электрона, определяемый зависимостью ширины области конфайнмента от напряженности электрического поля.

DOI: $10.21883 /$ OS.2018.09.46558.279-17

\section{Введение}

Системы низкой размерности характеризуются специфическим квантованием, что приводит к ряду новых явлений $[1,2]$. В частности, система пониженной размерности возникает при взаимодействии электрона, находящегося вблизи поверхности металла, с коллективом электронов внутри нейтрального проводника. Энергия этого взаимодействия зависит от расстояния до поверхности металла $z$. Статическая часть соответствующей энергии описывается в модели взаимодействия электрона с его изображением [3], в рамках которой электрон находится в одномерном кулоновском поле притяжения с потенциальной энергией $U=-1 / 4 z$ [4-6] (используется атомная система единиц: $\hbar=e=m=1$ ). Со стороны металла движение ограничено потенциальной стенкой. Такая модель соответствует „одномерному атому“ с эффективным зарядом ядра $Z=1 / 4$ и уровнями связанных состояний, подобных состояниям электрона в водородоподобном атоме с ридберговскими энергиями $E_{n}=-0.85 \mathrm{eV} /(n+a)^{2}$, где квантовый дефект $a$ слабо зависит от квантового числа уровня $n$ [7-9]. При этом в одномерной системе в отличие от трехмерной вырождение уровней отсутствует. Теоретически состояния в одномерном потенциале изображения описаны в работе [10], а экспериментально они наблюдались при двухфотонной резонансной эмиссии электронов из металла [11-13].

Последовательный подход к описанию связанных состояний вблизи поверхности металла основан на много- частичном механизме электронного взаимодействия [14]. Однако влияние нелокальных многоэлектронных эффектов на высоковозбужденные состояния невелико, и модель потенциала изображений для них вполне пригодна при описании движения электрона в электростатическом внешнем поле. Действие внешнего однородного электрического поля на электрон, связанный потенциалом изображения, приводит к сдвигу энергетических уровней - эффекту Штарка. Этот эффект обнаруживается в измерениях энергий связи с использованием туннельного ионизационного микроскопа в сравнении с энергиями, получаемыми по результатам фотоэмиссии [15].

В рамках модели потенциала изображения ранее были получены точные волновые функции электрона, находящегося в потенциале изображения [16], и в первом порядке теории возмущений по внешнему однородному электрическому полю рассчитана поправка к энергии электрона (линейный эффект Штарка) [17]. Для более сильных полей отклик электрона, связанного в потенциале изображения, на электрическое поле был рассмотрен в квазиклассическом приближении, что позволило рассчитать энергии и волновые функции связанных состояний электрона вблизи поверхности металла вне рамок теории возмущений [18]. Все полученные ранее результаты относились к случаю, когда внешнее однородное электрическое поле направлено так, что оно вырывает электрон из связанного состояния. Целью настоящей работы является определение зависимости положения уровней энергии от величины внешнего однородного электрического поля произвольной напряженности, 
однонаправленного с полем изображения, и описание характеристик одномерного конфайнмента электрона в такой системе.

\section{Классические точки поворота}

Стационарное уравнение Шредингера для электрона в поле потенциала изображения и однородном электрическом поле, направленном перпендикулярно поверхности металла по оси $z$, имеет вид

$$
\left(\frac{\hat{p}_{x}^{2}+\hat{p}_{y}^{2}+\hat{p}_{z}^{2}}{2}-\frac{\alpha}{4 z}+F z-E_{t}\right) \psi_{1}(x) \psi_{2}(y) \psi_{3}(z)=0 .
$$

Здесь слагаемое $F z$ гамильтониана описывает энергию взаимодействия с электрическим полем напряженностью $F$, слагаемое $-\alpha / 4 z$ есть потенциальная энергия взаимодействия электрона с полем изображения. Константа $\alpha$ позволяет учесть дополнительные поверхностные эффекты, а в пренебрежении ими равна единице.

Переменные в уравнении (1) разделяются, так что компоненты волновой функции $\psi_{1}(x), \psi_{2}(y)$, совпадают с собственными функциями операторов проекций импульса $\hat{p}_{x}, \hat{p}_{y}$, и описываются плоскими волнами $\exp \left(i p_{x} x\right)$ и $\exp \left(i p_{y} y\right)$. Составляющая волновой функции $\psi(z) \equiv \psi_{3}(z)$ является решением уравнения

$$
d^{2} \psi(z) / d z^{2}+2\left(E_{z}-F z+\alpha / 4 z\right) \psi(z)=0,
$$

а полная энергия электрона определяется выражением

$$
E_{t}=p_{x}^{2} / 2+p_{y}^{2} / 2+E .
$$

Будем искать решение уравнения (2) в квазиклассическом приближении. В классически доступных областях движения величина

$$
p(z)=\sqrt{2(E-U(z))}
$$

совпадает с $z$-проекцией импульса электрона. Прямому применению квазиклассического приближения препятствует кулоновская сингулярность, но эта трудность преодолевается введением в потенциальную энергию поправки Лангера $[19,20]$. С учетом этой поправки потенциальная энергия принимает вид

$$
U(z)=F z-\alpha / 4 z+3 /\left(32 z^{2}\right) .
$$

Характерная зависимость потенциальной энергии $U(z)$ от координаты $z$ для полей разных направлений и напряженностей показана на рис. 1.

Рис. 1 демонстрирует наличие в физически доступной области $z>0$ (при противоположных направлениях поля изображения и внешнего однородного электрического поля) трех классических точек поворота (КТП). В этом случае есть две классически доступные области финитного $\left(z_{1} \leq z \leq z_{2}\right)$ и инфинитного $\left(z>z_{3}\right)$ движений. В случае совпадения направления полей имеются

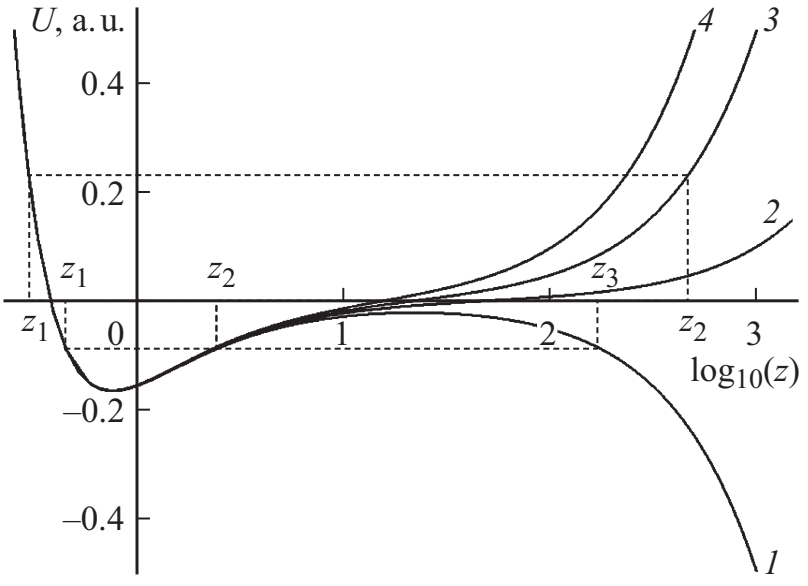

Рис. 1. Зависимость потенциальной энергии $U(z)$ электрона от расстояния до поверхности металла $z$ (а.u.) во внешнем электрическом поле различного направления и напряженности. Масштаб по оси $z$ логарифмический. Кривая 1 показывает потенциальную энергию электрона для поля с напряженностью $10^{-4}$ a.u., направленном противоположно полю потенциала изображения $(F<0)$. Кривые $2,3,4$ описывают потенциальные энергии для полей с напряженностями соответственно $10^{-4}$, $5 \cdot 10^{-4}, 10^{-3}$ a.u., направленными одинаково с полем потенциала изображения $(F>0)$.

лишь две точки поворота, и реализуется квантованное финитное движение, соответствующее одномерному конфайнменту электрона.

Определим размер области конфайнмента, ограниченной двумя КТП. Положение левой КТП $z_{1}$ в основном определяется сингулярными в нуле слагаемыми потенциальной энергии (5) - полем изображения и поправкой Лангера. Вследствие этого координата левой КТП практически не зависит от направления и модуля напряженности электрического поля. Поэтому ширина классически доступной области и квантование энергии определяются положением правой КТП $z_{2}$. Можно выделить три области параметров с качественно различной зависимостью положения правой КТП от энергии электрона и напряженности электрического поля.

1. При $E<-\sqrt{F}$ величина $z_{2}$ определяется кулоновским потенциалом и поправкой Лангера при слабой зависимости от напряженности внешнего электрического поля.

2. При малых $F$ в окрестности $E=0$ с шириной $\Delta E \sim 2 \sqrt{F}$ потенциал медленно растет с увеличением $z$, а протяженность классически доступной области значительно меняется при малых вариациях энергии.

3. При $E>\sqrt{F}$ потенциал $U(z)$ при больших значениях $z$ полностью определяется внешним электрическим полем, и размер классически доступной области линейно растет с увеличением $F$.

Опишем аналитически зависимость положения точек поворота от напряженности внешнего поля. Значения координат КТП являются положительными корнями 
уравнения

$$
F z+\alpha / 4 z-3 /\left(32 z^{2}\right)+E=0,
$$

которые можно выразить в виде

$$
\begin{gathered}
z_{1}=\left|\sqrt{1 / 3 F+(2 E / 3 F)^{2}} \cos (|\varphi|+\pi / 3)-\right| E|/ 3 F|, \\
z_{2}=\left[\left[E+\sqrt{3 \alpha F+4 E^{2}} \cos (\varphi)\right] / 3 F\right. \\
z_{3}=\left[E-\sqrt{3 \alpha F+4 E^{2}} \sin (\varphi+\pi / 3)\right] / 3 F
\end{gathered}
$$

Параметр $\varphi$ в уравнениях (7)-(9) следующим образом зависит от энергии электрона и напряженности поля:

$$
\varphi=\cos \left[\frac{1}{3} \arccos \left(\frac{72 \alpha E F-81 F^{2}+64 E^{3}}{8\left(3 \alpha F+4 E^{2}\right)^{3 / 2}}\right)\right] .
$$

Для полей одинакового направления корень $z_{3}$ находится в физически недоступной области $z<0$. Вследствие этого квантование полностью определяется положением двух положительных значений координат КТП, определяемых уравнениями (7) и (8).

Исследуем зависимость положения левой КТП $z_{1}$ от напряженности внешнего поля и энергии электрона. С точностью до членов $\sim F^{2}$ ее положение можно записать в виде

$$
\begin{aligned}
z_{1} & \approx(C(6,1)-\alpha) / 8 E-\left[\alpha C(9,2) C(6,1)^{-1 / 2}\right. \\
& -C(3,2)] F /\left(64 E^{3}\right)+\left[\left(135 E^{2} C(1,2)\right.\right. \\
& \left.\left.+2 \alpha^{4} C(45,4)\right) C(6,1)^{-3 / 2}-2 \alpha C^{2}(6,1)\right] F^{2} /\left(512 E^{5}\right) .
\end{aligned}
$$

Здесь введено обозначение

$$
C(n, m)=n E+m \alpha^{2} .
$$

В пределе больших значений энергии $E \gg \alpha^{2}$ положение левой КТП определяется поправкой Лангера, не зависит от $\alpha$ и может быть рассчитано по формуле

$$
\begin{aligned}
z_{1} \approx & \sqrt{6} /(8 \sqrt{E})+3 F /(8 E)^{2} \\
& +15 \sqrt{6} F /\left(2048 E^{7 / 2}\right)+9 F^{3} /(4 E)^{5} .
\end{aligned}
$$

Как видно из уравнений (11) и (13), в экспериментально достижимых полях $F<10^{-4}$ координата $z_{1}$ практически не зависит от напряженности внешнего поля.

В противоположном пределе малых энергий электрона $E \leq \alpha^{2}$ положение левой КТП определяется параметром $f=F^{3} / C^{2}(6,1)$ и с точностью до членов $f^{3}$ имеет вид

$$
\begin{aligned}
\lim _{E \rightarrow 0} z_{1} \approx & 3 / 8 \alpha+27 f /\left(128 \alpha^{2}\right) \\
& +729 f^{2} /\left(2048 \alpha^{3}\right)+6561 f^{3} /\left(8192 \alpha^{4}\right) .
\end{aligned}
$$

Разложения (11) и (14) верны всюду, за исключением окрестности минимума потенциальной энергии $E_{\min }$. С точностью до членов, квадратичных по напряженности поля, получим

$$
\begin{aligned}
E_{\min } \approx \alpha & (1-2 \sqrt{\alpha}) / 6+3 F\left(\alpha^{3 / 2}-\sqrt{\alpha}+1\right) /(2 \alpha)^{2} \\
& -3^{3} F^{2}\left(2 \alpha^{3 / 2}-4 \sqrt{\alpha}+3\right) /(2 \alpha)^{5}
\end{aligned}
$$

При энергиях, близких к $E_{\min }$, положение классических точек поворота также с точностью до квадратичных по полю членов представляется в виде

$$
\begin{aligned}
z_{1,2} \approx & \left( \pm C^{1 / 2}(6,1)-\alpha\right) / 8 E+F[C(3,2) C(6,1) \\
& \left.\mp \alpha C(9,2) C(6,1)^{-1 / 2}\right] / 64 C(6,1) E^{3}+F^{2}\left[\left(135 E^{3}\right.\right. \\
& \left.+270 \alpha^{2} E^{2}+8 \alpha^{6}+90 \alpha^{4} E\right) C^{1 / 2}(6,1) \\
& \mp 2 \alpha C(6,1) C(9,4)] / E^{5} C(6,1) .
\end{aligned}
$$

Здесь верхние знаки относятся к левой, а нижние - к правой КТП.

Как следует из уравнений (11) и (14), значения $z_{1}$ всюду, за исключением окрестности $E_{\min }$, практически не зависят от напряженности электрического поля и монотонно уменьшаются с ростом $E$, стремясь к асимптотическому значению $z_{1} \approx\left(\sqrt{6 E+\alpha^{2}}-\alpha\right) / 8 E$. При энергии, близкой к $E_{\min }$, в соответствии с уравнением (16) положение левой КТП линейно зависит от напряженности поля $F$. В экспериментально достижимых полях выполняется условие $F<10^{-4}$, и зависимость от напряженности не проявляется. Слабая зависимость положения левой КТП от напряженности, конечно, определяется ее локализацией в области малых ( $<1$ a.u.) расстояний от поверхности металла, в которой доминирует кулоновское взаимодействие и короткодействующая поправка Лангера, а влияние внешнего поля мало.

Сложнее выглядит поведение правой точки поворота $z_{2}$, положение которой в однородном электрическом поле одного направления с полем изображения изменяется в широких пределах в зависимости от напряженности внешнего поля и энергии электрона. Этот факт качественно меняет квантование системы по сравнению с ранее исследованным случаем разнонаправленных полей [18]. Для однонаправленных полей при энергии электрона, превышающей величину $-\sqrt{F}$, электрическое поле не является малым возмущением. В интервале энергий $|E|<\sqrt{F}$ вклады обоих полей в квантование соизмеримы, а в области $|E|>\sqrt{F}$ вклад внешнего поля становится основным, что иллюстрирует рис. 2. Вне интервала $|E|<\sqrt{F}$ вклады внутреннего и внешнего полей в энергию системы отличаются более чем на десятичный порядок, а в интервалах $|E|>5 \sqrt{F}-$ более чем на два порядка. 


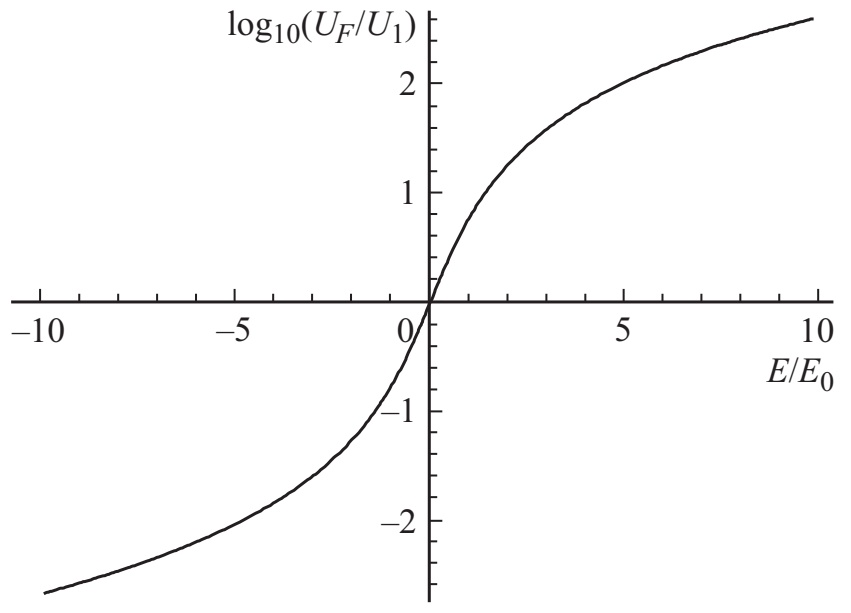

Рис. 2. Зависимость значения отношения потенциальной энергии электрона в правой КТП во внешнем электрическом поле $U_{F}=z F$ поля к сингулярной части потенциальной энергии $U_{1}=\alpha / 4 z-3 /\left(32 z^{2}\right)$ от полной энергии электрона. Масштаб по оси потенциальной энергии логарифмический, полная энергия нормирована множителем $E_{0}=\sqrt{F}$.

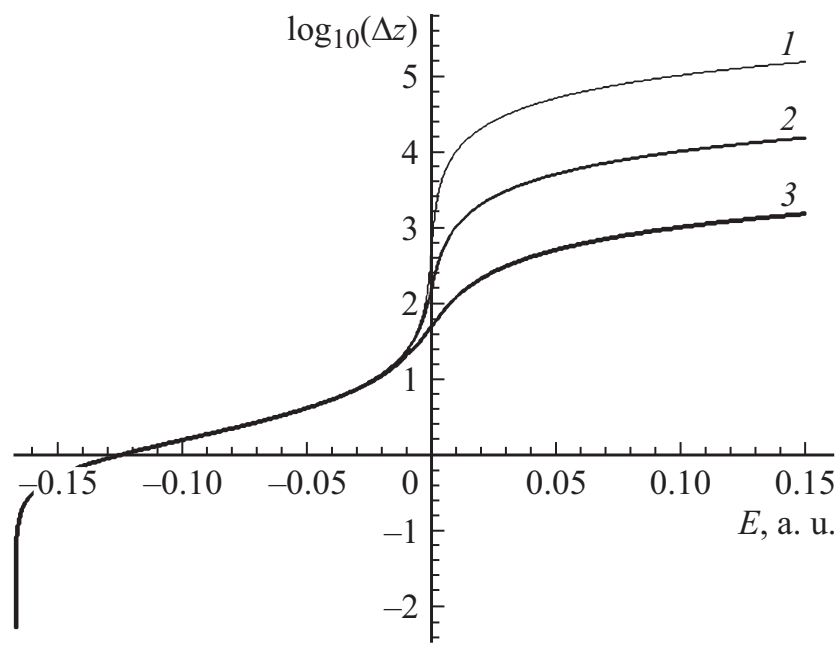

Рис. 3. Зависимость ширины области конфайнмента электрона $\Delta z$ от энергии электрона в полях $10^{-6}, 10^{-5}, 10^{-4}$ a.u. (кривые 1, 2,3).

Всюду, за исключением окрестности $E_{\min }$, ширина области конфайнмента электрона $\Delta z$ в экспериментально достижимых полях определятся положением правой КТП. В пределе $E \rightarrow E_{\min }$ классические точки поворота сливаются, и ширина $\Delta z$ стремится к нулю. Эту зависимость демонстрирует рис. 3, где наглядно проявляются две области с резко нелинейной зависимостью $\Delta z$. Первая область имеет ширину $\sim 2 \sqrt{F}$ при малых энергиях электрона. Вторая узкая область шириной $10^{-5}$ a.u. находится в окрестности минимума энергии. За пределами этих областей в интервалах энергий $E_{\min }+\sqrt{F}<E<-\sqrt{F}$ и $E>\sqrt{F}$ ширина зоны $\Delta z$ практически линейно зависит от внешнего поля и может быть описана в рамках теории возмущений по его напряженности.

\section{Квазиклассическое квантование}

Условие квантования может быть выражено через классическое действие

$$
S=\int_{z_{1}}^{z_{2}} p(z) d z
$$

в виде $[21,22]$

$$
S=(n+\mu) \pi,
$$

где поправка $\mu=-\sqrt{3} / 4$ выбрана из условия совпадения квазиклассического значения энергии с ее квантовомеханическим значением $E_{n}=-\alpha / 32 n^{2}$ в одномерном кулоновском потенциале $U=-\alpha / 4 z$ в отсутствие внешнего электрического поля.

Для дальнейшего анализа запишем классический импульс электрона через корни уравнения (6) в виде

$$
p(z)=\sqrt{2 F\left(z-z_{1}\right)\left(z_{2}-z\right)\left(z-z_{3}\right)} / z .
$$

Подставляя импульс из формулы (19) в уравнение (17), выразим действие $S$ через неполные эллиптические интегралы [23]:

$$
\begin{aligned}
& S=\frac{2}{3} \sqrt{\left(z_{1}-z\right)\left(z_{2}-z\right)\left(z_{3}-z\right)} \\
& +\frac{2}{3} \frac{\left(z_{3}^{2}-2 z_{1} z_{2}-z_{1} z_{3}-z_{2} z_{3}\right)}{\sqrt{z_{1}-z_{3}}} F\left(\sqrt{\frac{z-z_{1}}{z_{2}-z_{1}}}, \sqrt{\frac{z_{1}-z_{2}}{z_{1}-z_{3}}}\right) \\
& +\frac{2}{3} \sqrt{z_{1}-z_{3}}\left(z_{1}+z_{2}+z_{3}\right) E\left(\sqrt{\frac{z-z_{1}}{z_{2}-z_{1}}}, \sqrt{\frac{z_{1}-z_{2}}{z_{1}-z_{3}}}\right) \\
& +\frac{2}{\sqrt{z_{1}-z_{3}}} z_{2} z_{3} \operatorname{Pi}\left(\sqrt{\frac{z-z_{1}}{z_{2}-z_{1}}}, \frac{z_{1}-z_{2}}{z_{1}}, \sqrt{\frac{z_{1}-z_{2}}{z_{1}-z_{3}}}\right) .
\end{aligned}
$$

Подставив выражение (20) в формулу (18) и учитывая связь полных и неполных эллиптических интегралов [25], выразим условие квантования через положение классических точек поворота в виде

$$
\begin{aligned}
S= & \frac{2}{3} \sqrt{z_{1}-z_{3}}\left(z_{1}+z_{2}+z_{3}\right) E\left(\sqrt{\frac{z_{2}-z_{1}}{z_{1}-z_{3}}}\right) \\
& +\frac{2}{\sqrt{z_{1}-z_{3}}} z_{2} z_{3} \operatorname{Pi}\left(1, \frac{z_{1}-z_{2}}{z_{1}}, \sqrt{\frac{z_{2}-z_{1}}{z_{1}-z_{3}}}\right) \\
& +\frac{2}{3 \sqrt{z_{1}-z_{3}}}\left(z_{3}^{2}-z_{1} z_{3}-z_{2} z_{3}-2 z_{1} z_{2}\right) \\
& \times K\left(\sqrt{\frac{z_{1}-z_{1}}{z_{1}-z_{3}}}\right)=(n+\mu) \pi .
\end{aligned}
$$




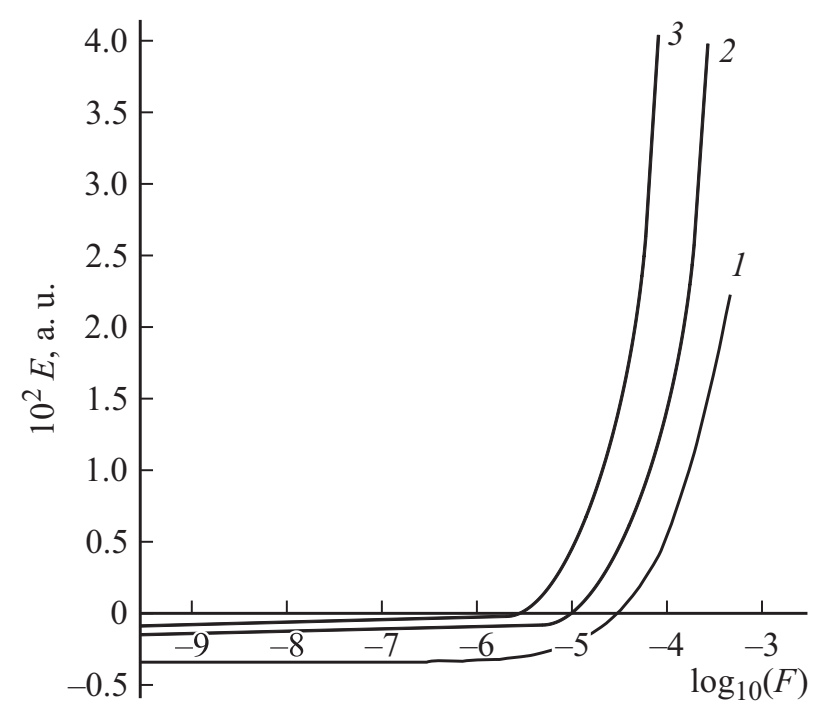

Рис. 4. Зависимость энергии состояний с квантовыми числами $n=3,5,7$ (соответственно кривые $1,2,3$ ) от напряженности электрического поля. Масштаб по оси абсцисс логарифмический.

Учитывая соотношения (7)-(9), запишем условие квантования через фазу (10) в виде

$$
\begin{aligned}
& S=\frac{2}{3} \sqrt{\frac{B \sin \varphi}{\sqrt{3} F}} \frac{E}{F} E(D) \\
& +\frac{2 \sqrt{\sqrt{3} F}}{3 \sqrt{B \sin \varphi}} \frac{2 E^{2}+3 F-2 E B \sin (\varphi+\pi / 6)}{6 F^{2}} K(D) \\
& +C \cdot \operatorname{Pi}\left(1, \frac{\sqrt{3} B \cos (\varphi+\pi / 6)}{B \cos (\varphi+\pi / 6)-E}, D\right)=(n+\mu) \pi .
\end{aligned}
$$

Здесь введены обозначения

$$
\begin{gathered}
B=\sqrt{3 F+4 E^{2}} \\
C=\frac{\sqrt{3} F[2 B E \cos (\varphi+\pi / 6)-}{9 F^{2}(B \sin \varphi)^{1 / 4}} \\
D=\sqrt{-\frac{\cos (\varphi+\pi / 6)}{\sin \varphi} .}
\end{gathered}
$$

Уравнение (22) позволяет определить зависимость энергии электрона, находящегося в состоянии с квантовым числом $n$, от напряженности поля. Результаты численного решения уравнения (22) показаны на рис. 4. Они позволяют выявить три характерные интервала напряженности поля с качественно различной зависимостью энергии электрона от $F$, границы которых определяются невозмущенным значением энергии $E_{n}$.

1. В слабых полях при соблюдении условия $F<E_{n}^{2}$ электрическое поле незначительно изменяет энергию электрона, поскольку ширина области конфаймента практически не зависит от энергии электрона и напряженности внешнего электрического поля.

2. В полях, напряженность которых превышает значение $F=E_{n}^{2}$, при фиксированном квантовом числе значение энергии электрона практически полностью определяется величиной поля. Такое поведение объясняется чувствительностью пространственной ширины $\Delta z$ области конфаймента к величине напряженности, что приводит к быстрому росту энергии связанных состояний электрона с увеличением $\Delta z$.

3. В переходной области $F \sim E_{n}^{2}$ энергия электрона в равной мере определяется как невозмущенным значением $E_{n}$, так и величиной внешнего поля.

Определим зависимость энергии электрона от квантового числа при $E>\sqrt{F}$, когда связанность состояния обеспечивается внешним электрическим полем. Действие $S$ в слабых полях выразим через элементарные функции, и условие квантования с точностью до линейных по напряженности поля членов принимает вид

$$
\begin{aligned}
& \frac{17 E^{3 / 2}}{24 F}+\frac{39 \alpha}{\sqrt{E}}-\frac{\sqrt{6}}{4} \arctan \left(\frac{\alpha}{\sqrt{6 E}}\right) \\
& -\frac{\sqrt{\alpha}}{8 \sqrt{E}} \ln \left(\frac{F \sqrt{6 E+\alpha^{2}}}{16 E^{2}}\right)+\left[\left(6 E+19 \alpha^{2}\right)\right. \\
& \left.+12\left(2 E+\alpha^{2}\right) \ln \left(\frac{F \sqrt{6 E+\alpha^{2}}}{16 E^{2}}\right)\right] \frac{F}{1024 E^{5 / 2}}=\frac{n \pi}{\sqrt{2}} .
\end{aligned}
$$

В рассматриваемой области энергий доминирующим членом в правой части уравнения (24) является слагаемое $17 E^{3 / 2} / 24 F$. Следовательно, в первом приближении энергия состояния с главным квантовым числом $n$ зависит от напряженности поля как

$$
E_{n}^{F}=(12 \sqrt{2} n \pi F / 17)^{2 / 3} .
$$

В области малых энергий при выполнении условия $E<\sqrt{F}$ действие $S$ не выражается через элементарные

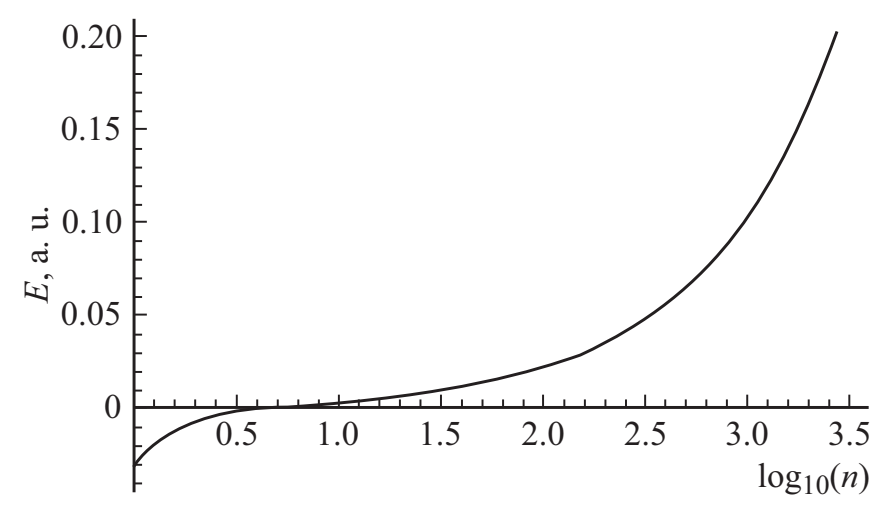

Рис. 5. Зависимость энергии электрона от квантового числа при напряженности электрического поля $F=10^{-5}$ a.u. Масштаб по оси абсцисс логарифмический. При $E>0.2$ a.u. зависимость $E(n)$ определяется формулой (25) с относительной ошибкой, не превышающей $1 \%$. 
функции. Решая численно уравнение (18), мы определим зависимость энергии от квантового числа. Примеры такой зависимости показаны на рис. 5, на котором резко выделяется интервал малых по модулю энергий $\Delta E=\lfloor-\sqrt{F}, \sqrt{F}\rfloor$ с высокой плотностью состояний. В поле напряженности $F=10^{-5}$ a.u. в этом энергетическом интервале находится $\sim 20$ состояний, и плотность состояний дискретного спектра равна $\sim 10^{4}$ (а.u. $)^{-1}$. Уменьшение напряженности поля приводит к быстрому росту плотности числа связанных состояний в интервале $\Delta E$. Так, в поле с напряженностью $F=10^{-10}$ a.u. плотность состояний $10^{6}$ (а.u. $)^{-1}$, а в поле $F=10^{-12}$ a.u. равна $10^{8}$ (а.u. $)^{-1}$. Такой быстрый рост плотности состояний дискретного спектра при малых энергиях электрона связан с быстрым увеличением ширины области конфайнмента при уменьшении напряженности электрического поля.

\section{Заключение}

Электрон, взаимодействующий с металлом, образует квазиодномерную кулоновскую систему, поведение которой во внешнем электрическом поле принципиально отличается от ее трехмерного варианта. Качественные особенности динамики такой системы определяются взаимным направлением напряженности постоянного электрического поля, перпендикулярной к поверхности металла, и напряженности поля изображения. При одинаковом направлении внутреннего и внешнего полей есть только две КТП, и электрон находится в потенциальной яме между ними. Возникает конфайнмент, и спектр энергий становится чисто дискретным. В экспериментально достижимых электрических полях положение левой точки поворота не зависит от напряженности поля. Фактически при любых энергиях электрона, за исключением узкой $(\sim \sqrt{F})$ окрестности минимума энергии, протяженность $\Delta z$ области конфайнмента определяется положением правой КТП, в зависимости от величины невозмущенной энергии электрона $E$. Значение $\Delta z$ монотонно растет с увеличением как $F$, так и $E$. Наиболее резкая зависимость $\Delta z(E)$ наблюдается в интервале энергий $|E| \leq \sqrt{F}$.

Квазиклассическое условие квантования позволяет определить уровни энергии в системе при наличии поля через полные эллиптические интегралы. В слабых полях при положительной энергии невозмущенного состояния электрона, удовлетворяющей условию $E>\sqrt{F}$, условие квантования выражается через элементарные функции. Поправка к величине классического действия определяется из условия совпадения квазиклассической энергии с точным квантово-механическим значением энергии в одномерном кулоновском потенциале в отсутствие электрического поля. Анализ численных значений рассчитанных энергий показывает, что при $E>-\sqrt{F}$ воздействие электрического поля на электрон не является малым возмущением. В поле с напряженностью $F>E_{n}^{2}$ энергия электрона определяется квантовым числом состояния и напряженностью поля, а взаимодействие с металлом может рассматриваться как возмущение. В переходной области энергий $|E| \leq \sqrt{F}$ необходимо одновременно учитывать вклад всех слагаемых потенциальной энергии в энергию электрона. В любом случае квантование энергии существенно определяется зависимостью ширины области конфайнмента электрона от квантового числа невозмущенного состояния и напряженности поля. Наиболее высокая плотность состояний дискретного спектра электрона наблюдается в энергетической области $|E| \leq \sqrt{F}$, характеризующейся быстрым уменьшением ширины области конфайнмента с ростом напряженности поля.

Экспериментальное измерение обсуждаемого нами конфайнмента электрона можно обеспечить, создавая запирающее поле с помощью наноиглы туннельного электронного микроскопа. Резонансное возбуждение связанных состояний вблизи поверхности металла эффективно осуществляется с помощью возбуждаемого фемтосекундным лазерным импульсом ближнего поля плазмонов, испытывающих на конце иглы сверхфокусировку [26]. Таким способом можно добиться локализации исследуемого эффекта. Для регистрации возбужденных состояний можно использовать их спонтанное излучение после окончания действия ультракороткого импульса.

В ходе дальнейших исследований целесообразно подробнее изучить поведение системы в интервале энергий $|E| \leq \sqrt{F}$, где наблюдается резкая зависимость ширины области конфайнмента от напряженности внешнего поля. Необходимо также установить зависимость волновой функции электрона от напряженности внешнего электрического поля и квантового числа состояния.

\section{Список литературы}

[1] Научные основы нанотехнологий и новые приборы / Под. ред. Р. Келсалла, А. Хэмли, М. Геогегана. Долгопрудный: Изд. Дом .Интеллект., 2011. 527 с.

[2] Harrison P. Quantum wells, wires and dots. Chichester: Wiley, 2010. $538 \mathrm{p}$.

[3] Ландау Л.Д., Лифиии, Е.М. Электродинамика сплошных сред. М.: Наука, 2002. С. 24.

[4] Echenique P.M., Uranga M.E. // Surface Sci. 1991. V. 247. P. 125.

[5] Echenique P.M., Berndt R., Chulkov E.V., Fauster T., Goldmann A., Höfer U. // Surface Sci. Rep. 2004. V. 52. P. 219.

[6] Еремеев С.В., Циркин С.С., Чулков Е.В. // ФТТ. 2010. Т. 52. C. 1644.

[7] Echenique P.M., Uranga M.E. // Surface Sci. 1991. Vol. 247. P. 125.

[8] Chulkov E.V., Silkin V.M., Echenique P.M. // Surface Science. 1997. V. 391. P. L1217.

[9] Chulkov E.V., Silkin V.M., Echenique P.M. // Surface Science. 1999. V. 437. P. 330. 
[10] Fauster Th., Reuß Ch., Shumay I.L., Weinelt M. // Chem. Phys. 2000. V. 251. P. 111. Fauster Th., Weinelt M. // J. Electron Spectroscopy and Related Phenomena. 2001. V. 1114-116. P. 269.

[11] Echenique P.M., Pendry J.B. // Prog. Surf. Sci. 1990. V. 32. P. 111-172.

[12] Shumay I.L., Höfer U., Reuß Ch., Thomann U., Wallauer W., Fauster Th. // Phys. Rev. B. 1998. V. 58. N 20. P. 13974.

[13] Fann W.S., Storz R., Bokor J. // Phys. Rev. B. 1991. V. 44. N 19. P. 10980.

[14] Echenique P.M., Berndt R., Chulkov E.V., Fauster Th., Goldman A., Höfer U. // Surface Sci. 2004. V. 52. P. 219.

[15] Chulkov E.V., Borisov A.G., Gauyacq J.P., Sánchet-Portal D., Silkin V.M., Zhukiv V.P., Echenique P.M. // Chem Rev. 2006. V. 106. P. 4160.

[16] Головинский П.А., Преображенский М.А. // Опт. и спектр. 2015. T. 118. № 2. С. 203.

[17] Головинский П.А., Преображенский М.A. // Письма в ЖТФ. 2015. Т. 41. № 15. С. 8.

[18] Головинский П.А., Преображенский М.A. // Оптика и спектроскопия. 2017. Т. 122, № 1. С. 133.

[19] Langer R. // Phys. Rev. 1937. V. 15. P. 669.

[20] Karnakov B.M., Krainov V.P. WKB approximation in atomic physics. Berlin: Springer, 2013. 176 p.

[21] Ландау Л.Д., Либиии, Е.М. Квантовая механика. Нерелятивистская теория. М: Наука, 1974. С. 339.

[22] Мигдал А.Б. Качественные методы в квантовой теории. М: Наука, 1975. 335 c.

[23] Abramowitz M., Stegun I. Handbook of Mathematical Functions. NY.: Dover Publications Inc., 1965. 1046 p.

[24] Bateman $H$. Higher transcendental functions. 1953. V. 1. NY: McGraw-Hill, 1955. 292 p.

[25] Fröman N., Fröman P.O. Stark effect in a hydrogenic atom or ion. Imperial College Press, 2008. 152 p.

[26] Мануйлович Е.С., Астапенко В.А., Головинский П.А. // Квант. электрон. 2016. Т. 46. № 1. С. 50. 